\title{
Prise en charge du cancer du rein de stade avancé : Mise à jour 2013 des lignes directrices consensuelles du Forum canadien sur le cancer du rein
}

\author{
Forum canadien sur le cancer du rein 2013
}

$\mathrm{N}$ ous présentons ici le quatrième rapport du Réseau canadien de recherche en cancer du rein (RCRCR); il comprend une mise à jour élaborée lors du quatrième Forum canadien sur le cancer du rein tenu en janvier 2013 à Toronto, en Ontario ${ }^{1-3}$.

Le cancer du rein, en particulier I'hypernéphrome, vient au premier rang des cancers génito-urinaires en fait de mortalité, entraînant le décès de plus de 1700 Canadiens chaque année 4 . Pour des raisons encore obscures, l'incidence globale de ce cancer augmente de $2 \%$ chaque année; la plupart des nouveaux cas concernent de petites masses rénales. Les traitements généraux ciblés, intégrés à la pratique clinique avec l'expérience croissante, existent depuis plus de 7 ans. La préservation de la fonction rénale grâce à l'adoption à grande échelle de la néphrectomie partielle est un élément central du traitement aux premiers stades de la maladie. Ces percées, ainsi que d'autres, ont transformé les soins et stimulé la recherche. Plusieurs lignes directrices au Canada traitent des différents aspects des soins chez les patients porteurs d'un hypernéphrome $e^{2,3,5,6}$.

Les trois forums précédents avaient eu lieu en 2008, 2009 et 2011. Comme les précédents, le forum de 2013 était de petite envergure et sur invitation; y assistaient des survivants du cancer et des soignants, de même que des cliniciens experts et des chercheurs dans le domaine du cancer du rein. Les participants incluaient des représentants de l'Association canadienne du cancer du rein ${ }^{7}$.

Pendant la conférence, les énoncés consensuels antérieurs ont été revus et mis à jour suivant le même processus. Le présent rapport est une mise à jour de la section portant sur la prise en charge du cancer de stade avancé du consensus publié en $2011^{3}$. Ici encore, le forum a abordé les points suivants : (1) stratégies de maîtrise du cancer du rein au Canada, qui incluent le Système d'information canadien sur le cancer du rein (SICCR), maintenant opérationnel; (2) l'élaboration d'une approche coordonnée pour la validation des lignes directrices proposées sur les tests génétiques pour les patients et les familles à risque de cancer du rein; (3) la promotion d'une sensibilisation accrue aux questions de survie liées au cancer, en particulier l'élaboration d'un plan de soins aux survivants; et (4) la poursuite du processus d'amélioration de la qualité afin de valider les indicateurs de qualité définis pour la prise en charge du cancer du rein. Les participants ont aussi discuté des modèles de prestation des tests génétiques et du counselling pour les patients atteints de cancer du rein, et de la nécessité de mettre à la disposition des patients à risque de présenter des cancers héréditaires les services dont ils ont besoin. Enfin, un certain nombre de nouvelles initiatives de recherche sur les soins contre le cancer du rein s'inscrivant dans la " médecine personnalisée » ont été mises de l'avant. Ces sujets seront présentés dans de futurs rapports. Le présent consensus porte sur la prise en charge du cancer de stade avancé. Un document distinct portant sur le cancer de stade précoce, y compris son diagnostic et la prise en charge chirurgicale, sera publié séparément.

\section{Prise en charge du cancer du rein localement avancé}

\section{Traitement néoadjuvant}

\section{Le traitement néoadjuvant avant une résection planifiée n'est} pas indiqué en dehors des essais cliniques.

Si on juge au moment du diagnostic qu'une résection chirurgicale est convenable, il est recommandé de procéder sans tarder à cette intervention. Le recours systématique aux traitements néoadjuvants n'est pas indiqué à ce moment. Les résultats finaux des essais cliniques sur les agents antiangiogéniques adjuvants et néoadjuvants (inhibiteurs de la tyrosine kinase du récepteur du facteur de croissance endothélial vasculaire [ITK du VEGFr], anticorps anti-VEGF ou inhibiteurs de la cible mammalienne de la rapamycine [mTOR]) ne seront pas publiés avant plusieurs années. 
Certains patients chez qui on juge une intervention chirurgicale non convenable au moment du diagnostic peuvent présenter une réponse marquée au traitement ciblé, et devront être réévalués par un urologue s'il y a une quelconque possibilité que la chirurgie soit devenue convenable.

\section{Traitement adjuvant}

\section{Le traitement adjuvant après résection chirurgicale n'est pas in- diqué, sauf dans le cadre d'un essai clinique.}

Le traitement adjuvant avec des cytokines n'améliore pas la survie globale après la néphrectomie ${ }^{8}$. Les résultats de plusieurs essais cliniques avec les agents antiangiogéniques adjuvants (ITK du VEGFr, anticorps anti-VEGF ou inhibiteurs de mTOR) ne seront pas publiés avant plusieurs années. Les patients porteurs de tumeurs à risque élevé ayant subi une résection complète devraient être invités à participer à des essais cliniques dans la mesure du possible.

\section{Cancer du rein avancé (métastatique)}

Il faut toujours envisager d'inscrire les patients à des essais cliniques bien conçus comme première option pour les patients atteints d'un hypernéphrome avancé ou métastatique.

\section{Traitement de première ligne}

- Le traitement ciblé est l'option à privilégier (Tableau 1).

- On peut aussi songer à la surveillance active, car certains patients présentent un cancer asymptomatique à évolution lente.

- Chez un nombre très restreint de patients, on peut envisager le recours à des doses élevées d'interleukine 2 (IL-2).

Le domaine du traitement général évolue rapidement, et les recommandations formulées dans le présent document reflètent les données publiées au moment où les participants à la conférence consensuelle ont tiré leurs conclusions. À mesure que de nouvelles données sont publiées, les options thérapeutiques changeront inévitablement.

L'hypernéphrome est une maladie hétérogène, et plusieurs facteurs pronostics peuvent aider les cliniciens à stratifier leurs patients en fonction du risque. Ces facteurs peuvent être de nature clinique, comme l'indice fonctionnel du patient, et comprendre des paramètres de laboratoire. Le premier des scores pronostics a été publié par Motzer et ses collaborateurs et servait à définir les critères d'inclusion ou à stratifier les patients en vue de leur inscription aux essais cliniques ${ }^{9}$. C'est pourquoi les recommandations thérapeutiques présentées au Tableau 1 et le texte qui suit diffèrent en ce qui a trait au niveau de risque du patient. Ce système d'établissement du pronostic a été élaboré à l'époque des cytokines. À l'époque des traitements ciblés, Heng et ses collaborateurs ont publié un score similaire, mais pas tout à fait identique, pour la stratification en fonction du risque, score qui peut s'appliquer à tous les patients recevant un traitement de nos jours ${ }^{10}$.

Selon les données d'études de phase III, le sunitinib entraîne des taux plus élevés de réponse, une meilleure qualité de vie (QdV) et une plus longue survie sans progression (SSP) que l'interféron alpha chez les patients atteints $\mathrm{d}^{\prime}$ un hypernéphrome métastatique à cellules claires ${ }^{11}$. Une analyse subséquente de la survie a révélé que les patients traités par sunitinib ont une survie globale (SG) plus longue que les patients traités par interféron ${ }^{12}$. De plus, des études de population provenant de la Colombie-Britannique et de I'Alberta ont montré des taux de survie globale presque doubles en présence d'un hypernéphrome métastatique depuis le lancement du sunitinib et du sorafénib ${ }^{13,14}$. La dose et le calendrier posologique du sunitinib doivent être optimisés en fonction de chaque patient afin d'obtenir le maximum de bienfait, ce qui peut nécessiter des ajustements par rapport au calendrier posologique standard consistant en quatre semaines de traitement suivies de 2 semaines sans traitement ${ }^{15}$. Selon les études de phase III, le pazopanib entraîne une prolongation de la survie sans progression (SSP) par rapport au placebo chez les patients jamais traités par cytokines ou réfractaires à ces dernières ${ }^{16}$. Comme traitement de première ligne, le pazopanib s'est aussi révélé non inférieur au sunitinib quant à la SSP dans l'essai COMPARZ (COMParing the efficacy, sAfety and toleRability of paZopanib vs. sunitinib) de phase III (information tirée seulement du résumé $)^{17}$. Un autre inhibiteur de la TK du VEGFr, le tivozanib, a entraîné une SSP supérieure par rapport au sorafénib dans une étude clinique de phase III menée auprès de patients atteints $d^{\prime} u n$ hypernéphrome à cellules claires qui n'avaient jamais été traités ou n'avaient pas reçu plus d'une ligne de traitement (à l'exception des ITK du VEGFr ou des inhibiteurs de mTOR) ${ }^{18}$.

D'après les données des études de phase III, le temsirolimus entraîne une prolongation de la SSP et de la SG par rapport à l'interféron seul ou à une association de temsirolimus et $d^{\prime}$ interféron chez les patients courant un risque plus élevé ${ }^{19}$. Un risque plus élevé était défini comme la présence d'au moins 3 des 6 critères suivants : indice de Karnofsky (indice KPS) de 60 à 70; hausse du taux de $\mathrm{Ca}++$; baisse du taux d'hémoglobine; hausse du taux de lactate déshydrogénase; $<1$ an entre la néphrectomie et le traitement, et multiples foyers métastatiques. Si l'accès aux médicaments est limité, l'évérolimus, $s$ 'il est offert, serait une option de rechange raisonnable ${ }^{20}$. Le pazopanib, le temsirolimus ou le sorafénib demeurent des options intéressantes chez les patients ne tolérant pas le sunitinib ${ }^{21}$.

Des données d'études de phase III montrent que I'association de bevacizumab et d'un interféron améliore davantage la SSP que l'interféron en monothérapie ${ }^{22,23}$. À 
I'heure actuelle, aucune soumission n'a été faite concernant l'emploi du bevacizumab dans le cancer du rein au Canada, et ce n'est donc pas une option pour les patients canadiens.

Les participants au forum croient qu'une période initiale de surveillance active est une option raisonnable chez certains patients, puisqu'aucun traitement général actuel n'offre la guérison, que tous les traitements offerts s'accompagnent $d^{\prime}$ effets secondaires potentiels et que certains patients présentent une maladie indolente accompagnée de métastases asymptomatiques à évolution lente.

Aucune étude de phase III portant sur I'usage de I'IL-2 n'a montré une amélioration de la survie, et cet agent n'est donc pas vu comme une norme thérapeutique, mais peut être envisagé chez des patients sélectionnés avec attention. Selon les études de phase II, cependant, un groupe très restreint de patients peut être considéré pour I'IL-2 ${ }^{24}$ à dose élevée. L'IL-2 à dose élevée doit être administrée dans des centres spécialisés et expérimentés et idéalement dans le cadre d'un essai clinique ou en contexte expérimental. II ne faut pas administrer l'IL-2 à faible dose ${ }^{25,26}$.

Chez les patients atteints d'un hypernéphrome métastatique ou avancé non à cellules claires, il faut encourager la participation à des essais cliniques. Parmi les autres options, notons le sunitinib, selon les analyses par sous-groupes de I'essai portant sur l'accès élargi et montrant son innocuité et son efficacité; le sorafénib, selon les analyses par sousgroupes de l'essai ARCCS (Advanced Renal Cell Carcinoma Sorafenib) portant sur l'accès élargi et montrant son innocuité et son efficacité, et le temsirolimus, selon I'analyse par sousgroupes des données des études de phase III27-30. Chez les patients atteints d'un hypernéphrome sarcomatoïde avancé ou métastatique ou d'un hypernéphrome mal différencié, les options incluent le sunitinib, en fonction d'études prospectives et sans randomisation du programme d'accès élargi; le sorafénib, selon l'étude ARCCS prospective et sans randomisation portant sur I'accès élargi; la chimiothérapie, en fonction des études de phase II portant sur des agents comme le 5FU, la gemcitabine, la doxorubicine et des associations de ces agents montrant une efficacité; et le temsirolimus, en fonction de l'analyse par sous-groupes de l'essai pivot de phase III auquel ces patients étaient admissibles ${ }^{27-29,31}$.

Au moment de prescrire un traitement général pour traiter un hypernéphrome avancé ou métastatique, plusieurs facteurs importants doivent être pris en compte. Un oncologiste connaissant les toxicités aiguës et à long terme, les interactions médicamenteuses, la façon de surveiller le traitement et la réponse au traitement devrait prescrire ce dernier. Les patients devraient être pris en charge par une équipe multidisciplinaire comprenant les ressources appropriées, dont une infirmière, une nutritionniste et un pharmacien. II faut évaluer fréquemment les patients afin de s'assurer de repérer les toxicités et de les traiter en conséquence. Patients et soignants devraient recevoir de l'information sur les effets secondaires potentiels, la prévention et le traitement.
Progression pendant un traitement par cytokines ou intolérance aux cytokines

Selon les études de phase III, le sorafénib a prolongé davantage la SSP par rapport aux meilleurs soins de soutien seuls chez des patients ayant déjà été traités par IL-2 ou interféron $^{32}$. Les données sur la SG ont été contaminées par la permutation mais ont atteint le seuil de signification lorsqu'elles étaient censurées pour éliminer l'effet de la permutation. Le pazopanib a également été étudié chez cette population de patients et entraîne une amélioration de la SSP par rapport au placebo ${ }^{16}$. L'axitinib a aussi entraîné une prolongation supérieure de la SSP par rapport au sorafénib dans cette population. Dans l'essai AXIS (axitinib vs. sorafenib in advanced RCC), environ un tiers des sujets avaient reçu des cytokines comme traitement de première ligne au moment de l'inscription à l'étude, et on a observé une prolongation de la SSP avec l'emploi de l'axitinib ${ }^{33}$. De même, le tivozanib a entraîné une SSP supérieure par rapport au sorafénib dans cette population ${ }^{18}$. Le sunitinib est une solution de rechange. Selon deux études de phase II, le sunitinib a amené des taux de réponse significatifs et a augmenté la survie sans progression en comparaison avec les témoins d'études antérieures ${ }^{34}$.

\section{Progression après le traitement ciblé de première ligne}

- II faut appuyer la conduite d'essais cliniques dans cette population, car la séquence optimale de traitement $n$ 'est pas connue.

- Passer à un autre agent ciblé (Tableau 1).

Selon les études de phase III, I'évérolimus (inhibiteur de mTOR par voie orale) a entraîné une SSP significativement plus longue que le placebo avec un profil de toxicité acceptable chez les patients n'ayant pas répondu au sunitinib ou au sorafénib (ou aux deux) ${ }^{35}$. Dans les cas où l'évérolimus n'est pas accessible, le temsirolimus ne doit pas être utilisé systématiquement comme agent de remplacement en raison des résultats inférieurs obtenus avec cet agent en comparaison avec le sorafénib dans cette population de patients, tel que démontré dans l'étude INTORSECT ${ }^{36}$.

Selon les données de I'essai de phase III AXIS, I'axitinib a entraîné une prolongation de la SSP en comparaison avec le sorafénib comme traitement de deuxième ligne chez les patients dont la maladie avait progressé après un traitement de première ligne avec le sunitinib et constitue de ce fait une autre option raisonnable comme traitement de deuxième ligne ${ }^{33}$.

À I'heure actuelle, aucune donnée ne permet de déterminer quel traitement de deuxième ligne présente des résultats supérieurs après un traitement par inhibiteur de la TK du VEGFr; par conséquent, l'évérolimus ou l'axitinib sont des options convenables.

Chez les patients atteints d'un hypernéphrome avancé ou métastatique n'ayant pas répondu au sunitinib ou au sorafé- 


\begin{tabular}{|c|c|c|c|}
\hline Contexte & Patients & $\begin{array}{c}\text { Thérapie } \\
\text { (données de niveau 1) }\end{array}$ & $\begin{array}{c}\text { Autres options } \\
\text { (données de niveau inférieur à 1) }\end{array}$ \\
\hline \multirow[t]{2}{*}{ Jamais traités } & Risque faible ou modéré & $\begin{array}{l}\text { Sunitinib } \\
\text { Bevacizumab+IFN* } \\
\text { Pazopanib } \\
\text { Tivozanib** }\end{array}$ & $\begin{array}{l}\text { IL-2 à dose élevée } \\
\text { Sorafenib } \\
\text { Surveillance active }\end{array}$ \\
\hline & Risque élevé & Temsirolimus & Sunitinib \\
\hline \multirow{3}{*}{ Deuxième ligne } & Réfractaires aux cytokines & $\begin{array}{l}\text { Sorafenib } \\
\text { Pazopanib } \\
\text { Tivozanib** } \\
\text { Axitinib }\end{array}$ & Sunitinib, bevacizumab+IFN* \\
\hline & $\begin{array}{l}\text { Recours antérieur à un traitement } \\
\text { ciblant le VEGF }\end{array}$ & $\begin{array}{l}\text { Évérolimus } \\
\text { Axitinib }\end{array}$ & Traitement ciblé non utilisé auparavant \\
\hline & $\begin{array}{c}\text { Recours antérieur à un inhibiteur } \\
\text { de mTOR }\end{array}$ & & ITK du VEGFr \\
\hline Troisième ligne ${ }^{* * *}$ & Tous les patients & & Traitement ciblé non utilisé auparavant \\
\hline \multicolumn{4}{|c|}{$\begin{array}{l}\text { IFN : interféron; IL-2 à dose élevée : interleukine } 2 \text { à dose élevée; VEGF: facteur de croissance endothélial vasculaire; ITK du VEGFr : inhibiteur de la tyrosine kinase du récepteur du VEGF; m } \\
\text { : cible mammalienne de la rapamycine. } \\
\text { *L'association bevacizumab + IFN n'est pas approuvée au Canada, mais a été approuvée aux États-Unis et en Europe. **À l'heure actuelle, le tivozanib n'a pas été homologué par Santé } \\
\text { Canada. ***A l'heure actuelle, aucun traitement de troisième ligne par voie orale n'a été approuvé par Santé Canada. }\end{array}$} \\
\hline
\end{tabular}

nib, les autres options incluent le passage à un autre ITK $\mathbf{d u}$ VEGFr (p. ex. du sunitinib au sorafénib ou du sorafénib au sunitinib) selon de nouvelles données montrant l'efficacité en traitement séquentiel ${ }^{37}$. Le rôle de l'interféron après un traitement ciblé n'est pas clair.

Dans le cas des patients dont le traitement de première ligne était un inhibiteur de mTOR, aucune donnée de niveau $1 \mathrm{n}$ 'a été obtenue permettant d'orienter les décisions quant aux traitements de deuxième ligne. Le recours à un ITK du VEGFr dans ce contexte est une option raisonnable, mais cette recommandation est formulée en fonction de données inférieures au niveau $1^{38}$.

À I'heure actuelle, Santé Canada n'a approuvé aucun agent comme traitement de troisième ligne. Cela dit, des données publiées étayent l'usage de traitements ciblés dans ce contexte. Dans I'essai RECORD-1 (Renal Cell cancer treatment with Oral RAD001 given Daily) comparant l'évérolimus à un placebo, $25 \%$ des sujets randomisés avaient reçu deux traitements par ITK du VEGFr avant l'inscription à l'étude et on a noté une prolongation significative de la SSP dans le groupe sous évérolimus ${ }^{20}$. Par conséquent, l'évérolimus serait une option raisonnable dans ce contexte.

\section{Rôle de la néphrectomie cytoréductrice}

\section{La néphrectomie cytoréductrice doit être envisagée chez des pa- tients porteurs d'un hypernéphrome métastatique bien sélectionnés.}

Les recommandations pour cette section reposent sur des données de niveau 1 provenant de patients traités par interféron. Les patients à qui la néphrectomie cytoréductrice convient bien incluent des patients porteurs d'une tumeur primitive pouvant être retirée par chirurgie et présentant un faible risque de morbidité périopératoire, des patients ayant un bon indice fonctionnel (indice ECOG de 0 ou 1), et des patients sans signe de métastase au cerveau ${ }^{25,37-38}$. Il est important de s'assurer que les patients qui doivent subir une néphrectomie cytoréductrice répondent à ces critères afin de maximiser les bienfaits, et pour s'assurer qu'il n'y a pas de possibilité de progression rapide de la maladie exigeant l'amorce immédiate d'un traitement général.

À l'heure actuelle, aucune donnée provenant d'études avec randomisation ne peut orienter la pratique concernant la néphrectomie cytoréductrice en fonction des traitements ciblés. Les décisions doivent être prises par extrapolation à partir (1) des données sur l'interféron; (2) des données rétrospectives d'études nord-américaines montrant une amélioration des résultats chez les patients ayant subi une néphrectomie cytoréductrice avant le recours à un traitement ciblé; (3) du fait que la plupart des patients (> $90 \%$ ) inscrits aux essais cliniques de phase III portant sur les ITK du VEGFr avaient déjà subi une néphrectomie cytoréductrice; et (4) en fonction du jugement clinique ${ }^{9,20,32,39-41}$. Des études prospectives sur les bienfaits de la néphrectomie cytoréductrice doivent être menés, et plusieurs essais sont en cours. Des chercheurs canadiens participent à l'essai SURTIME de I'EORTC (essai 30073).

Chez les patients qui ne subissent pas une néphrectomie cytoréductrice dès le départ mais répondent bien aux ITK du VEGFr ou à un traitement ciblé, qui présentent une atteinte métastatique limitée et un bon indice fonctionnel, la néphrectomie cytoréductrice peut être envisagée en cours de traitement. 


\section{Rôle de la métastatectomie}

Chez certains patients avec un nombre limité de foyers métastatiques et stables sur le plan clinique, une résection des métastases peut être raisonnable.

Aucun essai avec randomisation n'a montré les bienfaits de la métastatectomie dans le traitement de l'hypernéphrome. Cela dit, chez les patients porteurs de métastases métachrones après néphrectomie, environ un tiers est admissible à la métastatectomie; en fonction de plusieurs cohortes de grande taille, on rapporte un taux de survie de $50 \% 5$ ans après une résection complète des métastases ${ }^{37,42,43}$. En fonction des données d'observation dont on dispose, les patients les plus susceptibles de bénéficier d'une métastatectomie sont ceux qui ont reçu un diagnostic de métastases plus de 2 ans après la néphrectomie, ceux qui présentent des métastases isolées et ceux dont le siège des métastases est favorable. Il est raisonnable de laisser passer une période de surveillance active afin de confirmer le caractère indolent de la maladie métastatique.

\section{Rôle de la radiothérapie}

On peut envisager de recourir à la radiothérapie pour contrôler les saignements et la douleur liés à la tumeur primitive, pallier les symptômes des métastases et stabiliser les métastases au cerveau.

L'hypernéphrome n'est pas un cancer radiorésistant; de ce fait, bon nombre de patients peuvent obtenir une atténuation des symptômes du cancer grâce à la radiothérapie. Les nouvelles techniques, comme la radiothérapie stéréotaxique, peuvent améliorer les résultats par rapport à la radiothérapie externe conventionnelle; des essais sont actuellement en cours $^{44}$. Il faut appuyer la conduite d'essais sur la radiothérapie.

\section{Rôle des agents ciblant les os chez les patients présent- ant des métastases osseuses}

Environ un tiers des patients atteints d'hypernéphrome métastatique présenteront des métastases osseuses en lien avec leur tumeur principale ${ }^{45}$, ce qui peut entraîner des événements osseux. On a montré que les agents modificateurs du tissu osseux actuellement sur le marché réduisaient le taux d'événements osseux dans cette population. Dans un essai de phase III comparant l'acide zolédronique (AZ) au placebo, une analyse par sous-groupes comptant 74 patients atteints d'un hypernéphrome a montré que l'administration d'AZ entraînait une réduction significative des événements osseux par rapport au placebo (44\% contre $74 \%$ dans le groupe placebo ${ }^{46}$. Les résultats précis concernant ce sousgroupe ont été publiés séparément. On a observé une réduc- tion significative des événements osseux dans le groupe recevant de I'AZ à $4 \mathrm{mg}$ par voie intraveineuse une fois par mois en comparaison avec le placebo ${ }^{47}$. Par conséquent, I'administration mensuelle d'AZ est une option raisonnable. La surveillance attentive de la fonction rénale est requise. Les patients recevant des bisphosphonates courent le risque de présenter une hypocalcémie; la prise de suppléments de calcium et de vitamine $\mathrm{D}$ est donc recommandée. Cela dit, étant donné qu'une hypercalcémie paranéoplasique peut également se manifester en présence $d^{\prime}$ 'un hypernéphrome, la surveillance des taux sériques de calcium est primordiale. Il faut s'assurer que les patients qui entreprennent tout traitement ciblant les os ont subi un examen dentaire complet avant l'amorce du traitement et qu'ils font l'objet $d^{\prime}$ une surveillance continue afin de déceler l'apparition d'une ostéonécrose de la mâchoire.

Le dénosumab est un inhibiteur du RANKL (receptor activator of nuclear factor kappa-B ligand). Dans un essai de phase III comparant le dénosumab à l'acide zolédronique dans le traitement du cancer avec métastases osseuses (à l'exception du cancer du sein et du cancer de la prostate), un sous-groupe de patients inscrits à cet essai était atteint d'un hypernéphrome métastatique. Cet essai a montré la non-infériorité du dénosumab par rapport à l'AZ en ce qui a trait à la réduction des événements osseux dans le groupe pris dans son ensemble; par contre, aucune analyse par sousgroupes portant sur les patients atteints d'hypernéphrome n'a été menée $e^{48}$. À la lumière de ces données, on peut aussi considérer comme une option raisonnable le recours au dénosumab dans cette population de patients. La prise de suppléments de calcium et de vitamine D et une surveillance étroite des taux sériques de calcium sont aussi requises chez les patients sous dénosumab, de même qu'un examen dentaire complet et la surveillance des signes d'apparition d'une ostéonécrose de la mâchoire.

\section{Résumé}

De nombreuses percées ont pu être notées dans le traitement de l'hypernéphrome de stade avancé au cours des dernières années, avec l'ajout de plusieurs traitements ciblés dans l'arsenal thérapeutique. Le traitement doit être individualisé en fonction du niveau de risque du patient et le traitement par chaque agent choisi doit être optimisé quant à la dose et au calendrier posologique afin d'obtenir des bienfaits maximaux. La séquence optimale des agents reste à déterminer et fait l'objet d'essais cliniques en cours. Pour maximiser les bienfaits pour les patients, une approche multidisciplinaire revêt une importance capitale. Cela dit, malgré des percées récentes, on compte toujours de nombreux décès liés à l'hypernéphrome métastatique, et il est essentiel de continuer à appuyer les essais cliniques afin d'approfondir nos connaissances au sujet de cette maladie. 


\section{Références}

1. Management of kidney cancer: Canadian Kidney Cancer Forum Consensus Statement. Can Urol Assoc J 2008;2:175-82.

2. Management of kidney cancer: Canadian Kidney Cancer Forum Consensus Update. Can Urol Assoc J 2009;3:200-4.

3. Management of Kidney Cancer: Canadian Kidney Cancer Forum Consensus Update 2011. Can Urol Assoc J 2012;6:16-22.

4. Canadian Cancer Statistics 2012; 2012: Toronto, Ontario. http://www.cancer.ca/en/cancer-information/cancer-101/canadian-cancer-statistics-publication/. Accessed July 25, 2013.

5. Alberta Health Services, A.P.G.T.T., Renal cell carcinoma. Clinical Practice Guideline GU-0032011.

6. Kassouf W, Siemens R, Morash C, et al. Follow-up guidelines after radical or partial nephrectomy for localized and locally advanced renal cell carcinoma. Can Urol Assoc J 2008;3:73-6.

7. Kidney Cancer Canada. http://www.kidneycancercanada.ca/. Accessed July 25, 2013.

8. Messing $E M$, Manola J, Wilding $G$, et al. Phase III study of interferon alfa-NL as adjuvant for resectable renal cell carcinoma: an Eastern Cooperative Oncology Group/Intergroup trial. J Clin Oncol 2003;21:1214-22. http://dx.doi.org/10.1200/JC0.2003.02.005

9. Motzer RJ, Bacik J, Schwartz LH, et al. Prognostic factors for survival in previously treated patients with metastatic renal cell carcinoma. J Clin Oncol 2004;22:454-63. http://dx.doi.org/10.1200/ JC0.2004.06.132

10. Heng DY, Xie W, Regan MM, et al. Prognostic factors for overall survival in patients with metastatic renal cell carcinoma treated with vascular endothelial growth factor-targeted agents: results from a large, multicenter study. J Clin Oncol 2009;27:5794-9. http://dx.doi.org/10.1200/JC0.2008.21.4809

11. Motzer RJ, Hutson TE, Tomczak $P$, et al. Sunitinib versus interferon alfa in metastatic renal-cell carcinoma. N Engl J Med 2007;356:115-24. ttp://dx.doi.org/10.1056/NEJMoa065044

12. Motzer RJ, Hutson TE, Tomczak $P$, et al. Overall survival and updated results for sunitinib compared with interferon alfa in patients with metastatic renal cell carcinoma. J Clin Oncol 2009;27:3584-90. hittp:// dx.doi.org/10.1200/JC0.2008.20.1293

13. Heng DY, Chi KN, Murray N, et al. A population-based study evaluating the impact of sunitinib on overall survival in the treatment of patients with metastatic renal cell cancer. Cancer 2009;15:776-83. http:// dx.doi.org/10.1002/cncr.24051

14. Warren $M$, Venner $P$, North $S$, et al A population-based study examining the effect of tyrosine kinase inhibitors on survival in metastatic renal cell carcinoma in Alberta and the role of nephrectomy prior to treatment. Can Urol Assoc J 2009;3:281-9.

15. Biarnason $G$, et al. Effect of an individualized dose/schedule strategy for sunitinib in metastatic renal cell cancer (mRCC) on progression-free survival (PFS): Correlation with dynamic microbubble ultrasound (DCE-US) data. 2011 Genitourinary Cancers Symposium. J Clin Oncol 2011;29 (suppl 7; abstr 356).

16. Sternberg CN, Davis ID, Mardiak J, et al. Pazopanib in locally advanced or metastatic renal cell carcinoma: results of a randomized phase III trial. J Clin Oncol 2010;28:1061-8. http://dx.doi.org/10.1200/ JCO.2009.23.9764

17. Motzer RJ, Hutson TE, Reeves J, et al. Randomized, open label phase III trial of pazopanib versus sunitinib in first line treatment of patients with metastatic renal cell carcinoma (mrcc): results of the COMPARZ trial. ESMO 2012, Vienna, Austria.

18. Motzer RJ, Nosov D, Eisen T, et al. Tivozanib versus sorafenib as initial targeted therapy for patients with advanced renal cell carcinoma: Results from a phase III randomized, openlabel, multicenter trial. J Clin Oncol 2012;30(suppl; abstr 4501).

19. Hudes $G$, Carducci $M$, Tomczak $P$, et al. Temsirolimus, interferon alfa, or both for advanced renal-cell carcinoma. N Engl J Med 2007;356:2271-81. http://dx.doi.org/10.1056/NEJMoa066838

20. Motzer RJ, Escudier B, Oudard S, et al. Phase 3 trial of everolimus for metastatic renal cell carcinoma: final results and analysis of prognostic factors. Cancer 2010;116:4255-65. http://dx.doi.org/10.1002/ cncr.25219

21. Escudier B, Eisen T, Stadler WM, et al. Sorafenib in advanced clear-cell renal-cell carcinoma. N Engl J Med 2007;356:125-34. http://dx.doi.org/10.1056/NEJMoa060655

22. Escudier $B$, Pluzanska $A$, Koralewski $P$, et al. Bevacizumab plus interferon alfa-2a for treatment of metastatic renal cell carcinoma: a randomized, double-blind phase III trial. Lancet 2007;370:2103-11. http://dx.doi.org/10.1016/S0140-6736(07)61904-7

23. Rini, B.I., et al., Bevacizumab plus interferon alfa compared with interferon alfa monotherapy in patients with metastatic renal cell carcinoma:CALBG 90206. J Clin Oncol 2009. 26(33): p. 5422-5428. http:// dx.doi.org/10.1200/JC0.2008.16.9847

24. Cancer Care Ontario. Interleukin-2 in the treatment of patients with unresectable or metastatic renal cell cancer. Evidence based series 3-8-2, version 2. September 2011.

25. Cancer Care Ontario Genitourinary Cancer Practice Guidelines. https://www.cancercare.on.ca/toolbox/ qualityguidelines/diseasesite/genito-ebs/. Accessed July 25, 2013.
26. Negrier S, Perol D, Ravaud A, et al. Medroxyprogesterone, interferon alfa-2a, interleukin 2, or combination of both cytokines in patients with metastatic renal carcinoma of intermediate prognosis: results of a randomized controlled trial. Cancer 2007;110:2468-77. http://dx.doi.org/10.1002/cncr.23056

27. Gore ME, Szczylik C, Porta C, et al. Safety and efficacy of sunitinib for metastatic renal-cell carcinoma: an expanded-access trial. Lancet Oncol 2009;10:757-63. http://dx.doi.org/10.1016/S14702045(09)70162-7

28. Stadler WM, Figlin RA, McDermott DF, et al. Safety and efficacy results of the advanced renal cell carcinoma sorafenib expanded access program in North America. Cancer 2010;116:1272-80. http:// dx.doi.org/10.1002/cncr.24864

29. Dutcher JP, de Souza P, McDermott D, et al. Effect of temsirolimus versus interferon-alpha on outcome of patients with advanced renal cell carcinoma of different tumor histologies. Med Oncol 2009;26:202-9. http://dx.doi.org/10.1007/s12032-009-9177-0

30. Choueiri TK, Plantade A, Elson P, et al. Efficacy of sunitinib and sorafenib in metastatic papillary and chromophobe renal cell carcinoma. J Clin Oncol 2008;26:127-31. http://dx.doi.org/10.1200/ JC0.2007.13.3223

31. Nanus DM, Garino A, Milowsky MI, et al. Active chemotherapy for sarcomatoid and rapidly progressing renal cell carcinoma. Cancer 2004;101:1545-51. http://dx.doi.org/10.1002/cncr.20541

32. Escudier B, Eisen T, Stadler WM, et al. Sorafenib for treatment of renal cell carcinoma: Final efficacy and safety results of the phase III treatment approaches in renal cancer global evaluation trial. I Clin Oncol 2009;2710:3312-8. http://dx.doi.org/10.1200/JC0.2008.19.5511

33. Rini Bl, Escudier B, Tomczak $P$, et al. Comparative effectiveness of axitinib versus sorafenib in advanced renal cell carcinoma (AXIS): a randomised phase 3 trial. Lancet 2011;3;378:1931-9.

34. Motzer RJ, Basch E. Targeted drugs for metastatic renal cell carcinoma. Lancet 2007;370:2071-3. http:// dx.doi.org/10.1016/S0140-6736(07)61874-1

35. Motzer RJ, Escudier B, Oudard S. Efficacy of evirolimus in advanced renal cell carcinoma:a double blind, randomized, placebo-controlled phase III trial. Lancet 2008;372:449-56. http://dx.doi. org/10.1016/50140-6736(08)61039-9

36. Hutson T, Escudier B, Esteban E, et al. Temsirolimus vs. Sorafenib as Second Line Therapy in Metastatic Renal Cell Carcinoma: Results from the INTORSECT Trial. 2012 ESMO Meeting, Vienna, Austria.

37. Patard JJ, Pignot G, Escudier B, et al. ICUD-EAU international consultation on kidney cancer 2010: treatment of metastatic disease. Eur Urol 2011;60:684-90. http://dx.doi.org/10.1016/i.eururo.2011.06.017

38. Grünwald V, Weikert $S$, Seidel $C$, et al. Efficacy of sunitinib re-exposure after failure of an mTOR inhibitor in patients with metastatic RCC. Onkologie 2011;34:310-4. http://dx.doi.org/10.1159/000328575

39. Flanigan RC, Mickisch G, Sylvester R, et al. Cytoreductive nephrectomy in patients with metastatic renal cancer: a combined analysis. J Urol 2004;171:1071-6. http://dx.doi.org/10.1097/01. ju.0000110610.61545.ae

40. Mickisch GH. Urologic approaches to metastatic renal cell carcinoma. Onkologie 2001;24:122-6. http:// dx.doi.org/10.1159/000050298

41. Choueiri TK, Xie W, Kollmannsberger C, et al. The impact of cytoreductive nephrectomy on survival of patients with metastatic renal cell carcinoma receiving vascular endothelial growth factor targeted therapy. J Urol 2011;185:60-6. http://dx.doi.org/10.1016/i.juro.2010.09.012

42. Eggener SE, Yossepowitch 0 , Pettus JA, et al. Renal cell carcinoma recurrence after nephrectomy forlocalized disease: predicting survival from time of recurrence. J Clin Oncol 2006;24:3101-6. http://dx.doi. org/10.1200/JC0.2005.04.8280

43. Breau RH, Blute ML. Surgery for renal cell carcinoma metastases. Curr Opin Urol 2010;20:375-81. http://dx.doi.org/10.1097/MOU.0b013e32833c7ada

44. Stinaver MA, Kavanagh BD, Schefter TE, et al. Stereotactic body radiation therapy for melanoma and renal cell carcinoma: impact of single fraction equivalent dose on local control. Radiat Oncol 201 1;6:34. http://dx.doi.org/10.1186/1748-717X-6-34

45. Sahi C, Knox JJ, Clemons M, et al. Renal Cell Carcinoma Bone Metastases: Clinical Advances. Ther Adv Med Oncol 2010;2:75-83. http://dx.doi.org/10.1177/1758834009358417

46. Rosen $\mathrm{LS}$, Gordon D, Tchekmedyian $\mathrm{S}$, et al. Zoledronic acid versus placebo in the treatment of skeletal metastases in patients with lung cancer and other solid tumors: a phase III, double-blind, randomized trial-The Zoledronic Acid Lung Cancer and Other Solid Tumor Study Group. J Clin Oncol 2003;21:3150-7. http://dx.doi.org/10.1200/JC0.2003.04.105

47. Lipton A, Colombo-Berra A, Bukowski RM, et al. Skeletal complications in patients with bone metastases from renal cell carcinoma and therapeutic benefits of zoledronic acid. Clin Cancer Res 2004;10:6397S-403S.

48. Henry DH, Costa L, Goldwasser F, et al. Randomized, double-blind study of denosumab versus zoledronic acid in the treatment of bone metastases in patients with advanced cancer (excluding breast and prostate cancer) or multiple myeloma. J Clin Oncol 2011;29:1125-32. http://dx.doi.org/10.1200/ JC0.2010.31.3304 